\title{
CROSS-LAYER PERFORMANCE EVALUATION OF IP-BASED APPLICATIONS RUNNING OVER THE AIR INTERFACE
}

Dmitri Moltchanov, Yevgeni Koucheryavy, and Jarmo Harju

Tampere University of Technology, Institute of Communications Engineering, P.O.Box 553, FIN-33101, Tampere, Finland. \{moltchan,yk,harju\}@cs.tut.fi

\begin{abstract}
We propose a novel cross-layer analytic approach to performance evaluation of delay/loss sensitive IP-based applications running over the wireless channels. We firstly extend the small-scale propagation model representing the received signal strength to IP layer using the cross-layer mapping. Then, we replace the resulting IP layer wireless channel model by an artificial equivalent arrival process using the error/arrival mapping. To get performance parameters of interest we use this process together with arrival process modeling the traffic source as an input to the queuing system with deterministic service time, limited number of waiting position and non-preemptive priority discipline representing the IP packet service process of the wireless channel.
\end{abstract}

Keywords: IP layer wireless channels model, cross-layer mapping, performance evaluation.

\section{INTRODUCTION}

To predict performance of wireless channels, propagation models representing the received signal strength are often used. However, these models cannot be directly used in performance evaluation studies and must be previously extended to the IP layer at which QoS is defined. For such extension to be accurate, we have to take into account specific peculiarities of underlying layers including modulation schemes at the physical layer, data-link error concealment techniques and segmentation procedures between different layers. Wireless channel model for performance evaluation studies must be cross-layer complex function of propagation characteristics at the layer of interest.

Up to date only a few studies devoted to IP layer performance of applications running over the air interface have been published. Most studies were devoted to performance of the data-link layer protocols (Krunz and Kim, 2001; Zorzi et al., 1997) and rely on approaches specifically developed for wireless trans- 
mission medium. Hence, they often require new approximations, algorithms, stable recursions etc. Additionally, most of them adopt restrictive assumptions regarding the performance of wireless channels at layers above physical which may lead to incorrect estimation of IP layer performance parameters.

In this paper we propose a cross-layer analytic approach to performance evaluation of delay/loss sensitive IP-based applications running over the wireless channels. To use results available in queuing theory we show how to represent the problem of unreliable transmission over the wireless channel using a simple queuing model with two inputs. To achieve this goal we firstly extend the small-scale propagation model representing the received signal strength to the IP layer using the cross-layer mapping. Then, we replace the IP layer wireless channel model by an artificial equivalent arrival process using the error/arrival mapping. We use this process together with the arrival process modeling the traffic source as an input to the queuing system with deterministic service time, limited number of waiting position and non-preemptive priority discipline modeling the service process of the wireless channel.

Our paper is organized as follows. In Section 2 we review propagation characteristics of wireless channels and models used to capture them. In section 3 we propose an extension to the IP layer. Performance evaluation is carried out in Section 4. Then, in Section 5 we provide numerical examples. Conclusions are drawn in the last section.

\section{WIRELESS PROPAGATION CHARACTERISTICS}

To represent performance of wireless channels propagation models are used. Usually, we distinguish between large-scale and small-scale propagation models (Rappaport, 2002). The latter ones capture characteristics of wireless channel on a finer granularity than large-scale ones and implicitly take into account movements of users over short travel distances (Saleh and Valenzuela, 1987; Durgin and Rappaport, 2000). To capture small-scale propagation characteristics we have to take into account the presence of LOS between the transmitter and a receiver. In the presence of dominant non-fading component the small-scale propagation distribution is Rician. As the dominant component fades away Rician distribution degenerates to Rayleigh one.

\section{Model of small-scale propagation characteristics}

Assume a discrete-time environment, i.e. time axis is slotted, the slot duration is constant and given by $\Delta t=\left(t_{i+1}-t_{i}\right), i=0,1, \ldots$. We choose $\Delta t$ such that it equals to the time to transmit a single bit at the wireless channel. Hence, the choice of $\Delta t$ explicitly depends on properties of the physical layer. 
Small-scale propagation characteristics are often represented by the stochastic process $\{L(n), n=0,1, \ldots\}$ modulated by the discrete-time Markov chain $\left\{S_{L}(n), n=0,1, \ldots\right\}, S_{L}(n) \in\{1,2, \ldots, M\}$ each state of which is associated with conditional probability distribution function of the signal strength $F_{L}(k \Delta f \mid i)(\Delta f)=\operatorname{Pr}\left\{L(n)=k \Delta f \mid S_{L}(n)=i\right\}, k=1,2, \ldots, N, i=$ $1,2, \ldots, M$, where $N$ is the number of bins to which the signal strength is partitioned and $\Delta f$ is the discretization interval (Zhang and Kassam, 1999; Swarts and Ferreira, 1999). Underlying modulation allows to capture autocorrelation properties of the signal strength process. Since it is allowed for the Markov process $\left\{S_{L}(n), n=0,1, \ldots\right\}$ to change the state in every time slot, every bit may experience different signal strengths.

Let $D_{L}$ and $\vec{\pi}_{L}=\left(\pi_{1}, \pi_{2}, \ldots, \pi_{M}\right)$ be the one-step transition probability matrix and stationary probability vector of $\left\{S_{L}(n), n=0,1, \ldots\right\}$ respectively. Parameters $M, D_{L}, F_{L}(k \Delta f \mid i)(\Delta f)$, must be estimated from statistical data (Zhang and Kassam, 1999; Swarts and Ferreira, 1999). For the ease of notation we will use $F_{L}(k \mid i)$ instead of $F_{L}(k \Delta f \mid i)(\Delta f)$.

\section{WIRELESS CHANNEL MODEL AT THE IP LAYER}

The small-scale propagation model of the received signal strength defined in the previous section cannot be directly used in performance evaluation studies and must be properly extended to the IP layer at which QoS is defined. To do so we have to take into account specific peculiarities of layers below IP including modulation schemes at the physical layer, data-link error concealment techniques and segmentation procedures between different layers.

In the following we define models of incorrect reception of the protocol data units (PDU) at different layers. For this purpose we implicitly assume that the appropriate PDUs are consecutively transmitted at corresponding layers.

\section{Bit error process}

Consider a certain state $i$ of the Markov chain $\left\{S_{L}(n), n=0,1, \ldots\right\}$. Since the probability of a single bit error is the deterministic function of the received signal strength (Rappaport, 2002), all values of $F_{L}(k \mid i)$ that are less or equal to a computed value of so-called bit error threshold $B_{T}$ cause bit error. Those values which are greater than $B_{T}$ do not cause bit error. Thus, each state of the Markov process $\left\{S_{L}(n), n=0,1, \ldots\right\}$ can be associated with the following 
bit error probability:

$$
p_{E, i}=\operatorname{Pr}\left\{E(n)=1 \mid S_{E}(n)=i\right\}=\sum_{k=1}^{B_{T}} \operatorname{Pr}\left\{L(n)=k \mid S_{L}(n)=i\right\}
$$

where $\{E(n), n=0,1, \ldots\}, E(n) \in\{0,1\}$ is the bit error process for which 1 denotes incorrectly received bit, 0 denotes correctly received bit, $\left\{S_{E}(n), n=\right.$ $0,1, \ldots\}$ is the underlying Markov chain of $\{E(n), n=0,1, \ldots\}$. Note that $\left\{S_{L}(n), n=0,1, \ldots\right\}$ and $\left\{S_{E}(n), n=0,1, \ldots\right\}$ are the same and $\vec{\pi}_{E}=\vec{\pi}_{L}$, $D_{E}=D_{L}$, where $D_{E}$ and $\vec{\pi}_{E}$ are one-step transition probability matrix and stationary distribution vector of $\left\{S_{E}(n), n=0,1, \ldots\right\}$ respectively. $B_{T}$ must be estimated based on a modulation scheme and other specific features of the physical layer utilized at a given wireless channel (Rappaport, 2002).

Denote by $d_{E, i j}(k)=\operatorname{Pr}\left\{E(n)=k, S_{E}(n)=j \mid S_{E}(n-1)=i\right\}, k=0,1$, the transition probability from state $i$ to state $j$ with correct and incorrect bit reception respectively. These probabilities are represented in a compact form using matrices $D_{E}(1)$ and $D_{E}(0), D_{E}(1)+D_{E}(0)=D_{E}$. The state from which the transition occurs completely determines the bit error probability. The state to which transition occurs is used for convenience of matrix notation.

\section{Frame error process without FEC}

Assume that the length of the frame is constant and equal to $m$ bits. Sequence of consecutively transmitted bits, denoted by gray rectangles, is shown in Fig. 1 , where $(l-1), l,(l+1)$ denote time intervals whose length equal to the time to transmit one frame; $k, i, j$, denote the state of $\left\{S_{E}(n), n=0,1, \ldots\right\}$ in the beginning of these intervals.

Consider the stochastic process $\{N(l), l=0,1, \ldots\}, N(l) \in\{0,1, \ldots, m\}$, describing the number of incorrectly received bits in consecutive bit patterns of the length $m$. This process is doubly-stochastic with underlying Markov chain $\left\{S_{N}(l), l=0,1, \ldots\right\}$ and can be defined via the bit error process.

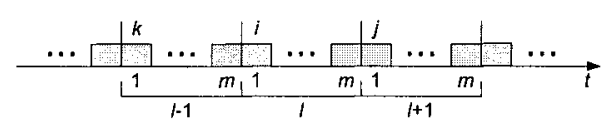

Figure 1. Sequence of consecutively transmitted bits at the wireless channel.

Denote the probability of going from state $i$ to state $j$ for the Markov chain $\left\{S_{N}(l), l=0,1, \ldots\right\}$ with exactly $k, k=0,1, \ldots, m$ incorrectly received bits in a bit pattern of length $m$ by $d_{N, i j}(k)=\operatorname{Pr}\left\{N(l)=k, S_{N}(l)=j \mid S_{N}(l-\right.$ 
1) $=i\}$. These probabilities can be found using $D_{E}(k), k=0,1, \vec{\pi}_{E}$ :

$$
\begin{aligned}
& d_{N, i j}(0)=\vec{\pi}_{E} D_{E}^{m}(0) \vec{e}, \\
& d_{N, i j}(1)=\vec{\pi}_{E} \sum_{k=m-1}^{0} D_{E}^{m-k-1}(0) D_{E}(1) D_{E}^{k}(0) \vec{e}, \\
& \ldots \\
& d_{N, i j}(m)=\vec{\pi}_{E} D_{E}^{m}(1) \vec{e},
\end{aligned}
$$

where $\vec{e}$ is the vector of ones of appropriate size.

Let us introduce the frame error process $\{F(l), l=0,1, \ldots\}, F(l) \in$ $\{0,1\}$, where 0 indicates the correct frame reception, 1 denotes incorrect frame reception. Process $\{F(l), l=0,1, \ldots\}$ is modulated by underlying Markov chain $\left\{S_{F}(l), n=0,1, \ldots\right\}$. Note that $\left\{S_{F}(l), l=0,1, \ldots\right\}$ and $\left\{S_{N}(l), l=\right.$ $0,1, \ldots\}$ are the same. Let us denote the probability of going from state $i$ to state $j$ for the Markov chain $\left\{S_{F}(l), l=0,1, \ldots\right\}$ with exactly $k, k=0,1$ incorrectly received frames by $d_{F, i j}(k)$. Process $\{N(l), l=0,1, \ldots\}$ describing the number of bit errors in consecutive frames can be related to the frame error process $\{F(l), l=0,1, \ldots\}$ using the so-called frame error threshold $F_{T}$ :

$$
d_{F, i j}(0)=\sum_{k=0}^{F_{T}-1} d_{N, i j}(k), \quad d_{F, i j}(1)=\sum_{k=F_{T}}^{m} d_{N, i j}(k) .
$$

Expressions (3) are interpreted as follows: if the number of incorrectly received bits in a frame is greater or equal to a computed value of the frame error threshold $\left(k \geq F_{T}\right)$ the frame is incorrectly received and $F(l)=1$, otherwise $\left(k<F_{T}\right)$ the frame is correctly received and $F(l)=0$.

Assume that FEC is not used at the data-link layer. It means that every time a frame contains at least one bit error, it is received incorrectly $\left(F_{T}=1\right)$. Thus, transition probabilities (3) of the frame error process take the following form:

$$
d_{F, i j}(0)=d_{N, i j}(0), \quad d_{F, i j}(1)=\sum_{k=1}^{m} d_{N, i j}(k)=1-d_{F, i j}(0) .
$$

The slot durations of $\{N(l), l=0,1, \ldots\}$ and $\{F(l), l=0,1, \ldots\}$ are the same $\Delta t^{\prime}$ and related to the slot duration of received signal strength process $\{L(n), n=0,1, \ldots\}$ as $\Delta t^{\prime}=n l \Delta t, n=0,1, \ldots$.

\section{Frame error process with FEC}

$F_{T}$ depends on FEC correction capabilities. Assume that the number of bit errors that can be corrected by a FEC code is $l$. Then, $F_{T}=(l+1)$ and a frame 
is incorrectly received when $k \geq(l+1)$. Otherwise, it is correctly received. Thus, transition probabilities (3) take the following form:

$$
d_{F, i j}(0)=\sum_{k=0}^{l} d_{N, i j}(k), \quad d_{F, i j}(1)=\sum_{k=l+1}^{m} d_{N, i j}(k) .
$$

\section{IP packet error process}

Assume that every IP packet is segmented into $z$ frames of equal size at the data-link layer. Sequence of consecutively transmitted frames, denoted by gray rectangles, is shown in Fig. 2, where $(h-1), h,(h+1)$ denote time intervals whose length equal to the time to transmit one packet; $k, i, j$, denote the state of the Markov chain $\left\{S_{F}(n), n=0,1, \ldots\right\}$ in the beginning of these intervals. Assumption of the constant frame size does not restrict the generality of results as long as only one traffic source is allowed to be active at any instant of time.

Let the stochastic process be $\{M(h), h=1,2, \ldots\}, M(h) \in\{0,1, \ldots, z\}$, describing the number of incorrectly received frames in a consecutive frame patterns of the length $z$. The process, modulated by Markov chain $\left\{S_{M}(h), h=\right.$ $0,1, \ldots\}$, can be completely defined via the frame error process.

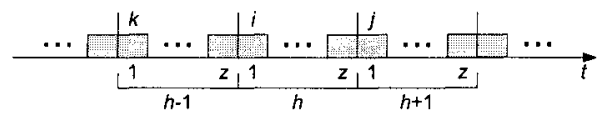

Figure 2. Sequence of consecutively transmitted frames at the wireless channel.

Denote the probability of going from state $i$ to state $j$ for the Markov chain $\left\{S_{M}(h), h=0,1, \ldots\right\}$ with exactly $k, k=0,1, \ldots, z$ incorrectly received frames in a frame pattern of length $z$ by $d_{M, i j}(k)=\operatorname{Pr}\left\{M(h)=k, S_{M}(h)=\right.$ $\left.j \mid S_{M}(h-1)=i\right\}$. These transition probabilities can be found using $D_{F}(k)$, $k=0,1$ and $\vec{\pi}_{F}$ of $\{F(l), l=0,1, \ldots\}$ as follows:

$$
\begin{aligned}
& d_{M, i j}(0)=\vec{\pi}_{F} D_{F}^{z}(0) \vec{e}, \\
& d_{M, i j}(1)=\vec{\pi}_{F} \sum_{k=z-1}^{0} D_{F}^{z-k-1}(0) D_{F}(1) D_{F}^{k}(0) \vec{e}, \\
& \ldots \\
& d_{M, i j}(z)=\vec{\pi}_{F} D_{F}^{z}(1) \vec{e},
\end{aligned}
$$

where $\vec{\pi}_{F}$ is the stationary distribution vector of $\left\{S_{F}(l), l=0,1, \ldots\right\}$.

Let us introduce the packet error process $\{P(h), h=0,1, \ldots\}, P(h) \in$ $\{0,1\}$, where 0 indicates correct packet reception, 1 denotes incorrect packet 
reception. Process $\{P(h), h=0,1, \ldots\}$ is modulated by underlying Markov chain $\left\{S_{P}(h), h=0,1, \ldots\right\} .\left\{S_{P}(h), h=0,1, \ldots\right\}$ and $\left\{S_{M}(h), h=\right.$ $0,1, \ldots\}$ are the same. Denote the probability of going from state $i$ to state $j$ for the Markov chain $\left\{S_{P}(h), h=0,1, \ldots\right\}$ with exactly $k, k=0,1$ incorrectly received packets by $d_{P, i j}(k)$. Process $\{M(h), h=0,1, \ldots\}$ describing the number of incorrectly received frames in consecutively transmitted packets can be related to the packet error process $\{P(h), h=0,1, \ldots\}$ using the so-called packet error threshold $P_{T}$ :

$$
d_{P, i j}(0)=\sum_{k=0}^{P_{T}-1} d_{M, i j}(k), \quad d_{P, i j}(1)=\sum_{k=P_{T}}^{z} d_{M, i j}(k)=1-d_{M, i j}
$$

Expressions (7) are interpreted as follows: if the number of incorrectly received frames in the packet are greater or equal to a computed value of the packet error threshold $\left(k \geq P_{T}\right)$ the packet is incorrectly received $(P(h)=1)$. Otherwise, it is correctly received $(P(h)=0)$. Since no error correction procedures are defined for IP layer, $P_{T}=1$ and only $d_{M, i j}(0)$ must be computed in (6). That is every time a packet contains at least one incorrectly received frame, the whole packet is received incorrectly.

Slot durations of $\{P(h), h=0,1, \ldots\}$ and $\{M(h), h=0,1, \ldots\}$ are the same $\Delta t^{\prime \prime}$ and related to the slot duration of the received signal strength process as $\Delta t^{\prime \prime}=n l h \Delta t, n=0,1, \ldots$.

\section{Illustration of the proposed extension}

An illustration of the proposed cross-layer mapping is shown in the Fig. 3 where time diagrams of $\{L(n), n=0,1, \ldots\},\{E(n), n=0,1, \ldots\}$, $\{N(l), l=0,1, \ldots\},\{F(l), l=0,1, \ldots\},\{M(h), h=0,1, \ldots\},\{P(h), h=$ $0,1, \ldots\}$ are shown. To define the model of incorrect reception of PDUs at different layers we implicitly assumed that appropriate PDUs are consecutively transmitted at corresponding layers. Hence, the IP packet error process is conditioned on the event of consecutive transmission of packets.

\section{PERFORMANCE EVALUATION}

In this subsection we provide an analytical technique for derivation of various performance parameters of the packet service process at the wireless channel. The proposed method is entirely based on classic queuing theory and applicable for delay/loss sensitive applications for which only FEC is implemented at the data-link layer. 


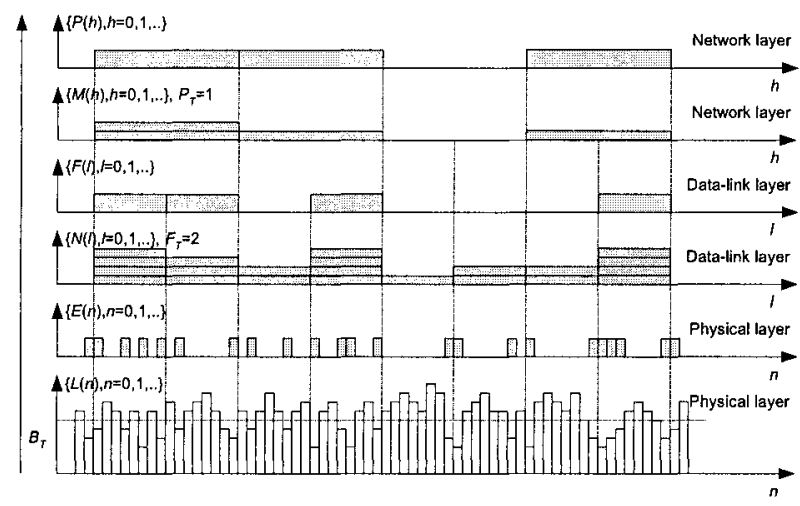

Figure 3. An illustration of the proposed cross-layer mapping.

Artificial arrival process. Given a Markov chain each state of which is associated with a certain packet error probability we propose to approximate the complexity of the packet error process by the so-called 'artificial' Markov modulated arrival process using error/arrival mappings. The mapping is straightforward: every time when the packet is incorrectly received with a certain probability, an arrival occurs with the same probability. Since it is not required to change the underlying Markovian structure and parameters of the IP layer wireless channel model, new parametrization procedure is not needed. The resulting arrival process is classified as a discrete-time Markovian arrival process (D-MAP). We denote it by $\mathrm{MAP}_{E}$.

Tagged arrival process. Assume that the arrival process from user's traffic source is represented by D-MAP $\left\{W_{T}(n), n=0,1, \ldots\right\}$ with underlying Markov chain $\left\{S_{T}(n), n=0,1, \ldots\right\}, S_{T}(n) \in\left\{1,2, \ldots, M_{T}\right\}$. According to it, each pair of states of the set $\left\{1,2, \ldots, M_{T}\right\}$ is associated with probability of one arrival, i.e. given a pair $(i, j), i, j=1,2, \ldots, M_{T}$ one arrival occurs in a slot with probability $p_{T, i j}=\operatorname{Pr}\left\{W_{T}(n)=1, S_{T}(n)=i \mid S_{T}(n-1)=i\right\}$ and no arrival occurs with complementary probability $1-p_{T, i j}$. We denote this process by $\mathrm{MAP}_{T}$

Description of the queuing system. We represent the service process of the wireless channel by the queuing system $\mathrm{MAP}_{T}+\mathrm{MAP}_{E} / \mathrm{D} / 1 / \mathrm{K}$ where arrivals coming from $\mathrm{MAP}_{E}$ are of higher priority than those ones coming from $\mathrm{MAP}_{T}$ and every arrival requires a service time of exactly one slot $\Delta t^{\prime \prime}$. The system operates as follows. At each slot boundary the server tries to serve an arrival from $\mathrm{MAP}_{E}$, if any. If not, it serves an arrival from $\mathrm{MAP}_{T}$. It is sufficient to assume a non-preemptive priority discipline for our system. Indeed, any arrival 
coming from $\mathrm{MAP}_{E}$ does not wait in the buffer and scheduled for service at the beginning of the nearest slot. Since at most one arrival may occur per a slot from $\mathrm{MAP}_{E}$ and these arrivals do not wait for service, waiting positions are only occupied by low priority arrivals. The service discipline is FCFS for low priority arrivals.

Queuing analysis. The loss probability is an important parameter for delay/loss sensitive applications. Given previously defined queuing model we have to determine packet losses experienced by $\mathrm{MAP}_{T}$ due to buffer overflow.

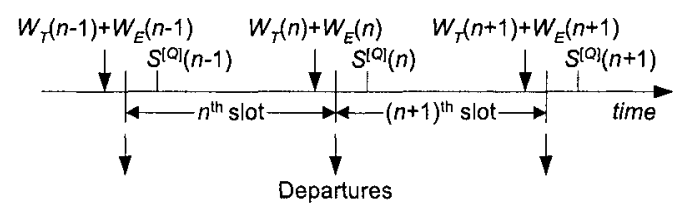

Figure 4. Time diagram of the queuing system.

Time diagram of the queuing system is given in the Fig. 4. Complete description of the queuing system requires a two-dimensional Markov chain $\left\{S_{A}(n), S_{Q}(n), n=0,1, \ldots\right\}$ embedded at the moments of departures, where $S_{A}(n)=S_{E}(n) \times S_{T}(n)$ is the state of superposition of $\mathrm{MAP}_{T}$ and $\mathrm{MAP}_{E}$, $S_{Q}(n) \in\{0,1, \ldots, K\}$ is the number of customers in the system at the moments of departures. Let $T$ be the transition matrix of such Markov chain and $\vec{x}=\left(x_{1,0}, . ., x_{M_{A}, K}\right), M_{A}=M_{E} M_{T}$ be the row array containing stationary probabilities. One can solve for $\vec{x}$ using $\vec{x} T=\vec{x}, \vec{x} \vec{e}=1$.

Let us determine the loss probability $L_{T}$ for $\mathrm{MAP}_{T}$ as a limiting probability of ratio between the number of lost arrivals of $\mathrm{MAP}_{T}$ in a slot and the number of arrivals from this process in a slot:

$$
L_{T}=\lim _{n \rightarrow \infty} \frac{L_{T}(n)}{W_{T}(n)}
$$

Since at most one arrival is allowed in a slot from each of arrival processes the loss of one customer from $\mathrm{MAP}_{T}$ always occurs when the state of the queuing system is $\left(S_{A}=i, S_{Q}=K\right), i=1,2, \ldots, M_{E} M_{T}$ and may occur when the state of the system is $\left(S_{A}=i, S_{Q}=K-1\right)$. We have:

$$
\begin{aligned}
L_{T}(n) & =\sum_{i=1}^{M_{E}} \sum_{j=1}^{M_{T}} x_{(i, j), K} \sum_{l=1}^{M_{T}} p_{T, j l}+ \\
& +\sum_{j=1}^{M_{E}} \sum_{i=1}^{M_{T}} x_{(i, j), K-1} \sum_{m=1}^{M_{E}} p_{E, i m} \sum_{l=1}^{M_{T}} p_{T, j l} .
\end{aligned}
$$


where $x_{(i, j), K}$ and $x_{(i, j), K-1}$ are $(i+j, K)$ and $(i+j, K-1)$ elements of stationary distribution of $\left\{S_{A}(n), S_{Q}(n), n=0,1, \ldots\right\}, p_{T, i j}, i, j=1,2, \ldots, M_{T}$ are probabilities of arrival from $\mathrm{MAP}_{T}$ going from state $i$ to state $j, p_{E, i j}$, $i, j=1,2, \ldots, M_{E}$ are probabilities of arrival from $\mathrm{MAP}_{E}$ going from state $i$ to state $j$. The mean arrival rate of $\mathrm{MAP}_{T}$ is given by:

$$
E\left[W_{T}\right]=\sum_{i=1}^{M_{T}} \pi_{T, i} \sum_{j=1}^{M_{T}} p_{T, i j},
$$

where $\pi_{T, i}$ and $\pi_{E, i}$ are elements of stationary probability vectors of $\left\{S_{T}(n), n=\right.$ $0,1, \ldots\}$ and $\left\{S_{T}(n), n=0,1, \ldots\right\}$ respectively. Substituting (10) and (9) in (8) we get a final expression for loss probability.

Let us now denote by $E\left[S_{T, Q}\right], E\left[S_{E, Q}\right]$ and $E\left[S_{A, Q}\right]$ the mean number of customers in the system of $\mathrm{MAP}_{T}, \mathrm{MAP}_{E}$ and superposed arrival processes respectively. For these quantities the following simple relation holds:

$$
E\left[S_{T, Q}\right]=E\left[S_{A, Q}\right]-E\left[S_{E, Q}\right]
$$

Since $\mathrm{MAP}_{E}$ has an absolute priority over $\mathrm{MAP}_{T}, E\left[S_{T, Q}\right]$ can be estimated from queuing system $\mathrm{MAP}_{E} / \mathrm{D} / 1 / \mathrm{K}$ neglecting those arrivals coming from $\mathrm{MAP}_{T}$. For arrivals from $\mathrm{MAP}_{E}$ in $\mathrm{MAP}_{E} / \mathrm{D} / 1 / \mathrm{K}$ queuing system the Little's result gives:

$$
E\left[S_{E, Q}\right]=E\left[W_{E}\right] E\left[D_{E}\right]
$$

where $E\left[D_{E}\right]$ is the mean sojourn time in the system of arrivals from $\mathrm{MAP}_{E}$. One may note that this time is always equal to the service time (one slot). Indeed, at most one arrival from $\mathrm{MAP}_{E}$ is allowed and this arrival is always served without waiting for service. Therefore, we have:

$$
E\left[S_{E, Q}\right]=\sum_{i=1}^{M_{E}} \pi_{E, i} \sum_{j=1}^{M_{E}} p_{E, i j}
$$

where $\pi_{E, i}$ is the $i^{\text {th }}$ element of steady-state distribution of $\left\{S_{E}(n), n=\right.$ $0,1, \ldots\} . E\left[S_{A, Q}\right]$ can be estimated from the stationary distribution $\vec{x}$ as:

$$
E\left[S_{A, Q}\right]=\sum_{i=1}^{M_{E} M_{T}} \sum_{k=0}^{K} x_{i, k} k .
$$

Finally, substituting (14) and (13) to (11) we get:

$$
E\left[S_{T, Q}\right]=\sum_{i=1}^{M_{E} M_{T}} \sum_{k=0}^{K} x_{i, k} k-\sum_{i=1}^{M_{E}} \pi_{E, i} \sum_{j=1}^{M_{E}} p_{E, i j}
$$




\section{NUMERICAL EXAMPLES}

To provide an example of IP layer performance evaluation let us assume that the wireless channel at the physical layer is represented by the Markov chain with $M=4, p_{E, i}=0, i=1,2,3, p_{E, 4}=0.01$ and the following transition probability matrix:

$$
P_{E}=\left(\begin{array}{cccc}
0.42 & 0.18 & 0.24 & 0.16 \\
0.18 & 0.42 & 0.04 & 0.36 \\
0.07 & 0.03 & 0.54 & 0.36 \\
0.03 & 0.07 & 0.09 & 0.81
\end{array}\right)
$$

Consider how the segmentation procedures between the data-link and IP layers affect the mean number of incorrectly received packets at the IP layer. Estimated values of the mean number of incorrectly received packets, $E[P]$, for different values of the number of frames to which the IP packet is segmented, $z$, number of bits in a single frame, $m$, number of incorrectly received bits that can be corrected, $l$, and Bernoulli arrival process with probability of one arrival set to 0.7 are shown in Fig. 5. Observing the obtained results we conclude that $z=1$ gives the best possible conditions at the IP layer. Note that setting $z=1$, $E[P]$ provides the number of incorrectly received frames at the data-link layer.
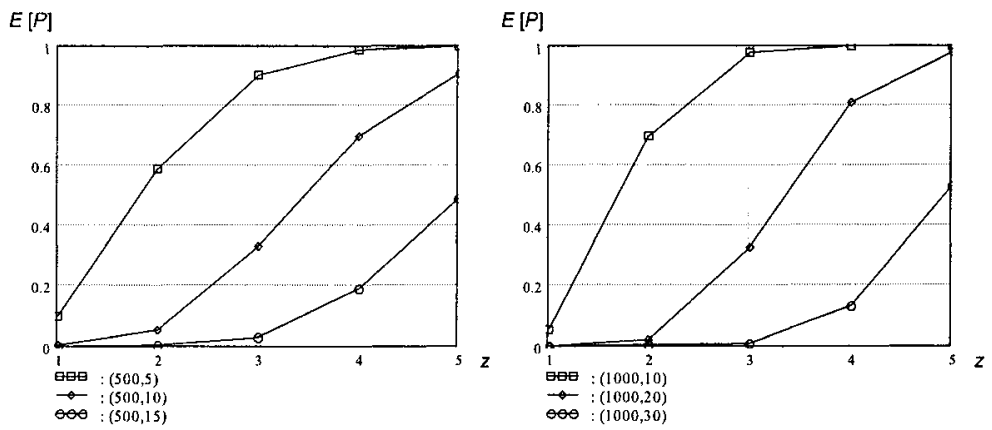

Figure 5. Mean number of lost IP packets for different values of $z, m$ and $l$.

Assume now that the wireless channel at the physical layer is represented by the Markov chain with $M=3, p_{E, 1}=0, p_{E, 2}=p_{E, 3}=1$, and the following transition probability matrix:

$$
P_{E}=\left(\begin{array}{ccc}
0.6 & 0.4 & 0 \\
0 & 0.2 & 0.8 \\
0.8 & 0 & 0.2
\end{array}\right) .
$$

We set $z=1$ and choose the arrival process from the traffic source to be Bernoulli with $p_{T}$ as the probability of one arrival. In the left part of the Fig. 
6 the packet loss probabilities are shown for different values of $p_{T}$ and different capacity of the system $K$. One may notice that given a wireless channel characteristics and $p_{T}>0.3$ packet losses become significant.

Let us now assume that it is possible to adjust parameters of environment such that $p_{E, 2}=0$. For example, it can done by proper displacement of the transmitter antenna. Loss probabilities for $p_{E, 2}=0$ and $p_{E, 2}=1$ are shown in the right part of Fig. 6. One may notice that the probability of loss decreases significantly when $p_{E, 2}=0$. For example, for $p_{T}=0.3$ and $K=9, L_{T}$ reaches $10^{-6}$.
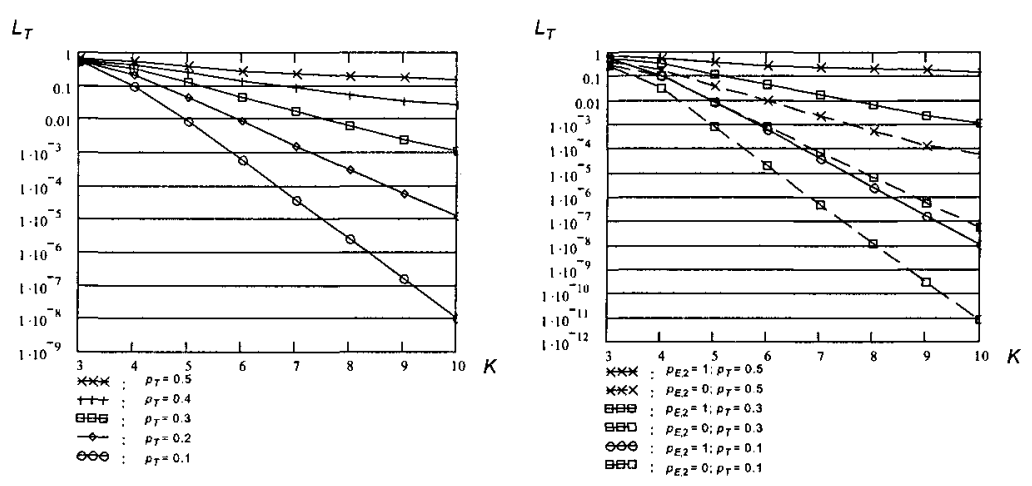

Figure 6. Loss probability $L_{T}$ for different $p_{T}$ and $p_{E, 2}$.

\section{CONCLUSIONS}

We provided a tool for accurate performance evaluation of delay/loss sensitive IP-based applications running over the wireless channels. The proposed wireless channel model can be used for performance evaluation at the IP layer using the classic queuing theory.

Despite of abovementioned advantages, the proposed model entirely relies on classic small-scale propagation model and does not take into account signal strength attenuation caused by movements over larger distances. The aim of our further work is to relax this assumption.

\section{REFERENCES}

Durgin, D. and Rappaport, T. (2000). Theory of multipath shape factors for small-scale fading wireless channels. IEEE Trans. on Ant. and Propag., 48:682-693. 
Krunz, M. and Kim, J.-G. (2001). Fluid analysis of delay and packet discard performance for QoS support in wireless networks. IEEE JSAC, 19(2):384-395.

Rappaport, T. (2002). Wireless communications: principles and practice. Communications engineering and emerging technologies. Prentice Hall, 2nd edition.

Saleh, A. and Valenzuela, R. (1987). A statistical model for indoor multipath propagation. IEEE JSAC, 5(2):128-137.

Swarts, J. and Ferreira, H. (1999). On the evaluation and application of markov channel models in wireless communications. In Proc. VTC'99, pages 117-121.

Zhang, Q. and Kassam, S. (1999). Finite-state markov model for Rayleigh fading channels. IEEE Trans. on Comm., 47(11):1688-1692.

Zorzi, M., Rao, R., and Milstein, L. (1997). ARQ error control for fading mobile radio channels. IEEE Trans, on Veh. Tech., 46(2):445-455. 